\title{
Acoustic synthesis of feed proteins under conditions of a complex ultrasonic field
}

\author{
Andrei Kochetov ${ }^{1}$, Valentin Minakov ${ }^{1}$, Elena Fisunova ${ }^{1}$, Tatyana Lavrenova, ${ }^{1, *}$, and Luybov \\ Filonenko ${ }^{1}$ \\ ${ }^{1}$ Don State Technical University, Gagarin square, 1, Rostov-on-Don, 344003, Russia
}

\begin{abstract}
Liquid waste of food industry enterprises contains various valuable organic substances of natural origin, which can be rationally used to obtain feed proteins. The problem of using valuable substances from food waste solves the problem of using biotechnological methods in order to use methods of accumulating biomass, enzymes, vitamins, etc. The biomass of unicellular microorganisms formed in the process of cultivation can be a raw material for obtaining feed additives and other biologically active substances.
\end{abstract}

\section{Introduction}

In recent years, obtaining new types of energy and fuel has become a priority problem for all countries of the world. Bioethanol (ethanol), one of the main types of biofuels, is obtained from sugar beets, sugarcane, corn, cereals, potatoes and other types of carbonaceous plant substrates. The global potential demand for bioethanol is 2 billion tons per year. Currently, the world produces 32 million tons of ethanol: of which 20 million tons - fuel ethanol, 8 million tons - ethanol for the chemical industry, 4 million tons - for the food industry. Chemical synthesis produces $7 \%$ ethanol, fermentation - 93\%. Obtaining alcohol by fermentation methods is accompanied by the formation of distillery bard during the production process. When alcohol (ethanol) is obtained from grain raw materials, more than $13 \mathrm{~m}^{3}$ per 1 ton of grain is formed. The problem of bard's disposal formed during the operation of factories producing alcohol is still an urgent problem. Most enterprises are trying to drain the unclaimed bard into nearby water bodies or fields, which seriously worsens the ecological situation around distilleries. The problem is compounded by the fact that bard is a perishable liquid (humidity is about $80 \%$ ) and at a temperature of $15-30^{\circ} \mathrm{C}$ it becomes contaminated with foreign microflora within a few hours [1].

There are three main technological schemes that are used in the processing of the bard:

- schemes with evaporation stations for obtaining "Dry bard (DDGS)";

- biogas production schemes;

- schemes for obtaining feed yeast.

The first group - schemes with evaporation plants to obtain "Dry bard (DDGS)". The technology of "centrate evaporation" in evaporation plants is the most widespread in the

*Corresponding author: bys ka87@mail.ru 
world. This technology is offered, for example, by the Swedish company Alfa-Laval, the Danish company Atlas-Stord, a number of Chinese and Russian companies (the so-called "Chinese scheme"), etc.

The second group - schemes with biogas production. Currently, these schemes have not found wide application either abroad or in Russia. The technology for processing bard into biogas is based on anaerobic fermentation (fermentation without oxygen). Almost all income from the sale of products goes to pay for energy resources, while the situation is getting worse every year. It should also be noted that in this production yeast assimilates only $20-30 \%$ of all dissolved organic substances from bard, although theoretically all dissolved organic substances are potentially assimilable, since they are all of natural origin. In principle, it is impossible to assimilate all organic matter of bard when using yeast-like fungi as producers due to their cultural and physiological characteristics (only a limited set of organic substances has the ability to assimilate) [2,3]. But such a problem can be solved using bacterial associations, an example of which is biological wastewater treatment, where deep removal of a wide variety of organic substances is achieved precisely when using mainly bacterial associations cultivated in aerotanks (the so-called activated sludge).

\section{Technological processes for obtaining feed proteins}

The first place in the production of BVK was taken by the United States, which accounts for more than $40 \%$ of the world production of soy protein. Russia produced substances of various vitamins, and now it is a country-importer. The positions of antibiotics' production are lost. The possibilities of the existing forage base of plant origin and, accordingly, the production of livestock products are limited. It is possible to increase the yield of meat products by processing liquid waste from food industry enterprises and raw materials of plant origin into fodder proteins on a new technological basis. The presented technological and technical solutions (section I of the book) make it possible to change the approach to the problem of processing waste from the food industry. New biotechnological equipment (fermenters), developed on the basis of the outlined solutions, will ensure the profitability of the process of processing waste from most food enterprises into fodder proteins. This is achieved due to the stability of the technological mode of operation of fermenters: longterm preservation of active biomass during breaks at work; the stability of the biomass of the packed part of the combustion chamber to temporary overloads and underloads on the organic substrate and the rapid recovery of biomass activity in the bubbling part with various deviations in the technological mode. In turn, chemical analytical control is simplified [4]. The process rationality is increased due to a 2-3-fold reduction in energy consumption and the reliability of the main equipment (low-pressure circulation pumps). The area on which the new equipment is located is significantly less than the area occupied by traditional biotechnological equipment, which allows it to be located in the limited areas of existing food production facilities. Finally, it becomes cost-effective to include biotechnological equipment for the production of fodder proteins in the equipment of poultry farms, pig farms and cattle farms. Fig. 1 shows the process flow diagram of the fermenter. 


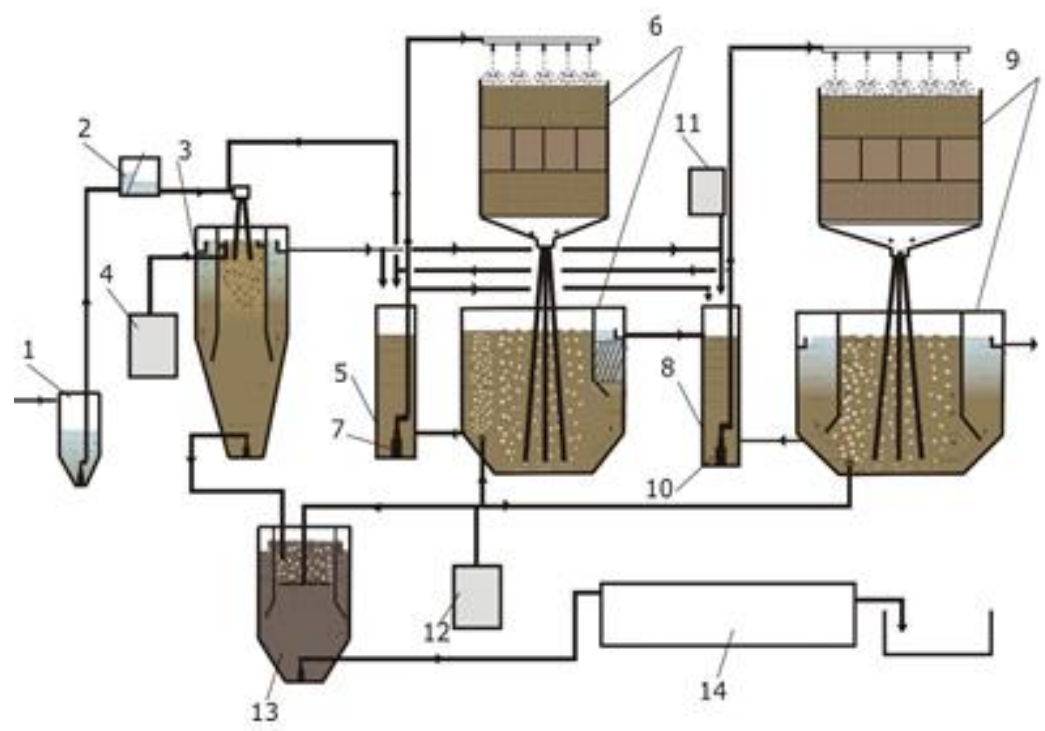

Fig. 1. Process flow diagram of the fermenter.

Liquid industrial waste from the shops goes to the receiving chamber of the pumping station (1), from where it is fed to the strainer (2). Further, the liquid is directed to the receiving chamber of the water-jet aerator of the biocoagulator-flotation device (3), where excess activated sludge is pumped from the compressor station automatically upon switching on the pumps in the pumping station. Due to the sorption properties of the removed sludge, suspended solids are precipitated by $50-70 \%$, dissolved organic contaminants are removed by the removed excess sludge (10-20\%), organic loads are partially averaged, $\mathrm{pH}$ and flotation separation of floating substances. The foam is discharged through the tray into the foam collector (4). The clarified liquid from the biocoagulator is directed to the mixing chamber (5) KS-1 (6), where, with the help of a circulation pump (7), the sludge mixture is repeatedly circulated through biofilters and aeration tanks with thin-layer modules.

Fig. 2 shows a diagram of the technological line for dewatering, granulation and sludge drying.

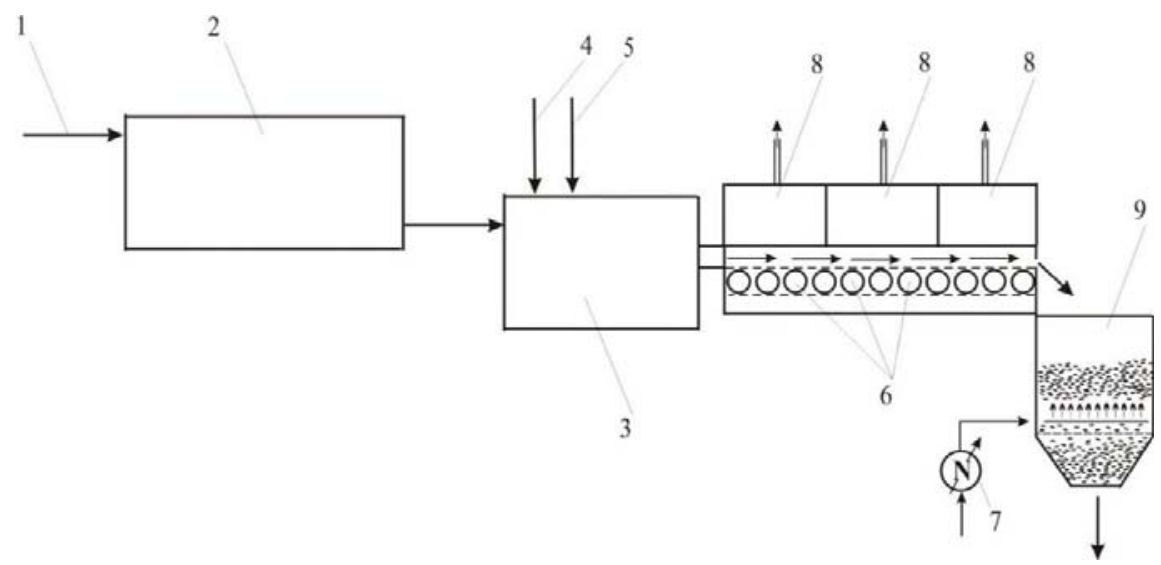

Fig. 2. Technological scheme for obtaining granular protein. 
According to the diagram, the pipeline for removing excess biomass (1) from the sludge compactor is connected to a screw dehydrator (2), where a cake with a given moisture content of $40-60 \%$ is formed. The dewatered cake is fed to the granulator (3). Dry waste of grain processing (bran) (4) and sludge from the filter (5) are fed there to reduce moisture and as a bonding structure of granules. Then the granules are fed onto the conveyor rollers (6). For rapid heating of granules, microwave emitters (8) with an exposure of 3 - 5 min were used. At the same time, there is a rapid drying and hardening of the granule shell due to its high adhesion ability. Next, the granules are fed into the storage tank (9) using a roller conveyor. The accumulators are equipped with devices for supplying and distributing warm air over the volume. For further drying of the granules for $6-10$ hours, air supply lines (7) from the workshops of the plant, pneumatic transport and heat pumps can be used. Due to the shock action of the mechanisms of dehydrators and granulators and the effect of microwave on the biomass, bacterial cells are damaged and enzymes are released. During the drying period, the enzymes facilitate the processing of bran (mainly fiber) and raw sludge from the strainer into a digestible form of protein. Next, the finished product is loaded onto the transport.

\section{The combined principle of mass transfer equipment existing solids of special shape by $7-10 \%$}

It is proposed to disperse fresh gas in apparatuses with a new principle of operation into a saturated solution of the withdrawn absorbent for the ultimate saturation of the liquid with gas molecules with the achievement of an equilibrium state and the complete completion of chemical reactions. At the same time, the final recovery of the lean gas components is carried out with fresh absorbent. Herewith, to create a large interface, use the acoustic dispersion of the absorbent solution into the smallest drops and drip irrigation using scattering reflectors. In a multistage scheme of mass transfer apparatus, use packed and bubble chambers with countercurrent and direct-flow gas and liquid movement. Use ceramic filling elements in packed chambers with the properties of rigid filling and disc-shaped charges. For the circulation of gas-liquid flows, use aeration columns with jet ejection of gases.

The design of the proposed apparatus using the technical solutions of the patent of the Russian Federation No. 2310499 from the application No. 2016114625 is shown in Fig.3. 


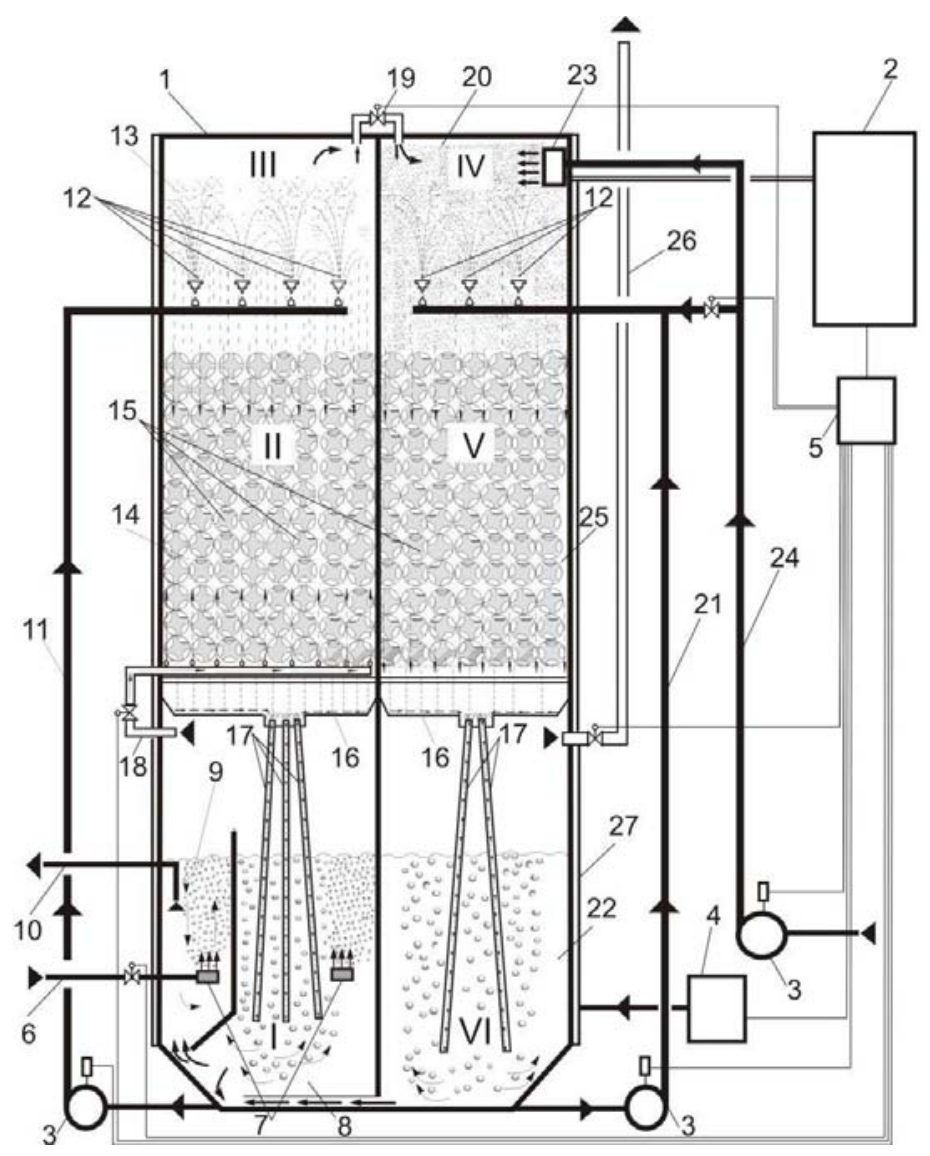

Fig. 3. Structural device of the mass transfer apparatus.

The device consists of a body with six chambers 1; acoustic dispersion module 2; circulation pumps 3 , air conditioner 4 and automatic control unit 5. Gas is fed through a pressure pipeline with a solenoid valve 6 to ceramic dispersers 7 and / or ultrasonic Hartmann whistles installed in the bubble chamber of the I stage of contact, which is divided by a partition into the main chamber 8 and the final one 9 A gas-liquid mixture of the circulating absorbent and gas is supplied to the chamber 8 through the aeration columns 17. In the chamber 8 , under intensive turbulent conditions, a high degree of solubility of the gas component in the absorbent and chemical reaction is achieved. Then the absorbent solution enters through the gap into the chamber 9. Dispersion of fresh gas bubbles using ceramic dispersers or a Hartmann whistle up to 500 microns and the residence time in the chamber ensure the achievement of the final equilibrium concentration of gas molecules in the solution. The saturated absorbent solution is discharged through pipeline 10. The incoming and circulating gases are mixed and accumulated in the interchamber space, where when the aeration columns are deepened 1$1.5 \mathrm{~m}$ below the liquid level, a partial gas pressure of 4-2 MPa can be created. The gas is then passed through line 18 to the bottom of the packing chamber 14 .

With the help of an electromagnetic valve installed on the pipeline, the required partial gas pressure in the interchamber space, the irrigation chamber 13 and the nozzle chamber 14. The gas entered into the chamber 14 is distributed through uniformly spaced nozzles 
throughout the chamber volume. The gas rising upward contacts the liquid flowing down the loading elements 15 . With countercurrent movement of gas and liquid through the spherical elements of the charge 15, a film of liquid and drops are formed on them. Through drops, a liquid film on the wetted surface (up to $80 \%$ of the total area of the packing element) and through the liquid surface in the recesses, the processes of further diffusion of the chemical components of the gas into the absorbent take place [5-8].

When filling the containers with one or two recesses in the spherical elements, the liquid flows over to the lower elements.

The saturation of the absorbent and the chemical reaction are increased by increasing the residence time of the liquid in the load.

Then the liquid flows down the collecting tray 16 into the drain collector and is discharged into the aeration columns 17, in which gas is sucked in and a gas-liquid mixture is formed. Gas bubbles at the exit from the lower ends of the columns separate and float. The estimated amount of gas entrained by the aeration columns at the height of the columns above the liquid of the bubble chamber 8 is within 1-2 $\mathrm{m}$ and the depths of the columns are $1-3 \mathrm{~m}$ is $0.9-0.5 \mathrm{~m}^{3} / \mathrm{m}^{3}$ of the liquid. At a partial gas pressure of chamber 14 above atmospheric $(0.1-4 \mathrm{MPa})$, the volume of gas sucked in by the columns increases. The highest values for the volume of entrained air and mass transfer coefficients are characteristic of aeration columns, in which well-developed vortex funnels are formed. The gas from the floating bubbles of the aeration columns 17 and bubbles of the dispersants 7 is discharged under pressure through the pipeline 18 into the packed chamber 14, and then into the irrigation chamber 13 . There, by the circulation pump 3 , through the pipeline 11 , the absorbent enriched with dissolved substances is supplied from the bubble chamber 22 and the liquid from camera 8.

When the liquid is supplied to the scattering reflectors 12 , the liquid flow is crushed against the cylindrical protrusions and slots of the reflective umbrellas (Fig. 4) As a result of which a large number of small, medium and large liquid droplets with different trajectories of movement are formed. Reflective disks according to RF patent No. 2422379 can also be used for crushing liquid.
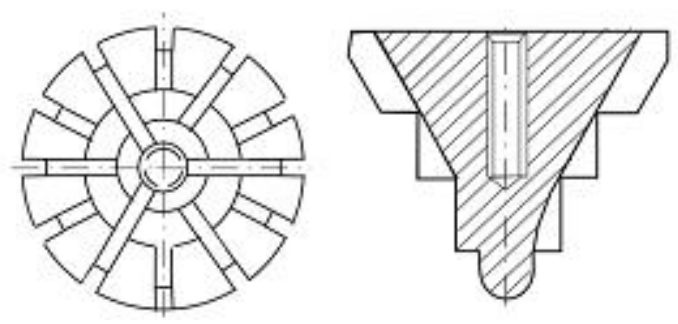

Fig. 4. Plan and section of a reflective umbrella with projections and longitudinal grooves.

Chamber 13 operates in the gas washer mode. Due to the increased partial gas pressure in the chamber 13, the gas flow moves through the pipeline 19 into the spray chamber 20 . The solenoid valve installed on the pipeline provides the calculated partial gas pressure in the chambers 20 and 25 .

The calculated effect of the extraction of gas components at the I stage is about $40-50 \%$ and $30-40 \%$ - at the II and III stages. In the mass transfer processes of the I, II and III stages, the absorption of volatile gas components is carried out. At the same time, a more difficult task is the absorption of substances from unreacted gas (10-30\%). Therefore, with the help of acoustic dispersion at the fourth stage of contact, the interfacial contact surface is significantly increased to trap hard-to-recover gas molecules. For this, a portion of the 
fresh absorbent of the order of $10-30 \%$ of the total consumption is fed into the chamber 20 to the acoustic disperser 23 through the pipeline 24 . The block diagram of module 2 with acoustic dispersant 23 (1) is shown in Fig. 5.

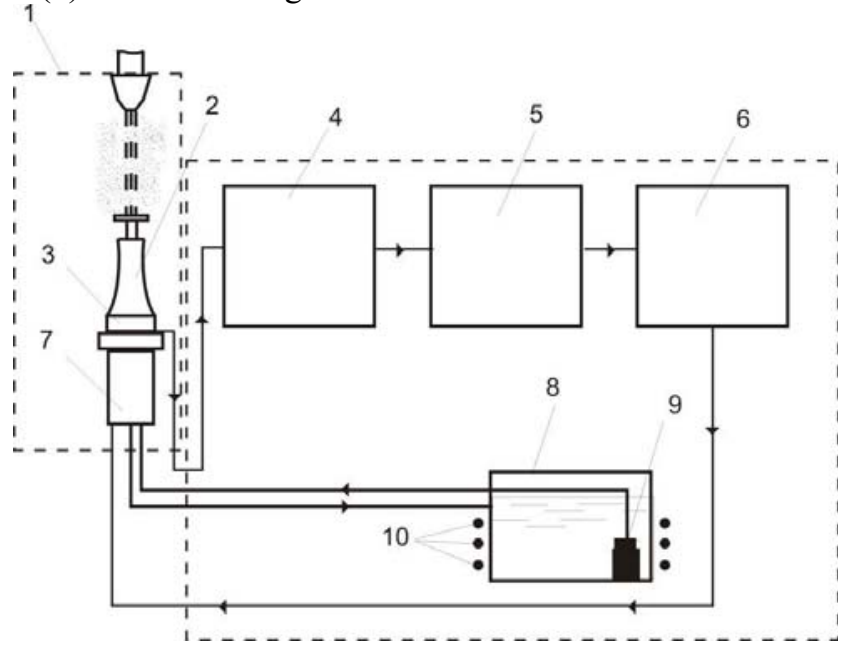

Fig. 5. Block diagram of the acoustic dispersant module.

In the scheme, the longitudinal-torsional ultrasonic waveguide 2 through the shaper of the acoustic feedback sensor 4 is connected to the module, which includes the automatic frequency control unit (AFC) 5, the ultrasonic generator USG-1-1M6, the inductive acoustic feedback sensor 3, which, in its turn, connected to the magnetostrictive transducer 7 (PMS1-1) of the waveguide 2. The module also includes a container 8 with a pump 9 and a cooling circuit 10 .

Acoustic dispersion is based on the effect of cavitation, which allows the development of large values of pressure arising from the collapse of cavitation cavities. In this case, the value of the pressure amplitudes can reach hundreds of thousands of atmospheres, which ensures the dispersion of liquid media. Due to the use of a longitudinal-torsional ultrasonic waveguide in the acoustic disperser 23, the dispersion coefficient is increased by 2 or more times. To achieve the maximum possible acoustic dispersion of the liquid, the ratio between the longitudinal and torsional components should be $1: 3$. This ratio is fulfilled at an angle of inclination channels with respect to the axis of rotation of the waveguide equal to $60^{\circ}$. The ratio of the areas of the ends of the waveguide provides a given transmission coefficient of the waveguide, which causes an oscillation of the acoustic system at the output of $15 \mu \mathrm{m}$ at an input amplitude of $10 \mu \mathrm{m}$. The waveguide has a resonant frequency of $22 \mathrm{kHz}$. An industrial model of a magnetostrictive transducer PMS1-1 (7), with an inductive acoustic feedback sensor 3 attached to its end, is used as an acoustic system on which the waveguide is fixed. Sensor 3 at the maximum amplitudes of oscillation of the speaker system has a maximum output voltage of the order of $1 \mathrm{~V}$ and a sinusoidal form of signal change in proportion to the frequency of the speaker system. The resonant frequency of the acoustic dispersion circuit is $22+0.1 \mathrm{kHz}$. The signal generated by the sensor 3 is fed to the shaper of the acoustic feedback sensor 4, which carries out digital filtering of the input signal, after which it is amplified and fed to the automatic frequency control unit 5, which performs active control of the servo system so that the vibration amplitudes at the output of the ultrasonic generator 6 was maximum. The ultrasonic generator USG 1-1 converts electrical energy of industrial frequency $50 \mathrm{~Hz}$ into ultrasonic energy $22 \mathrm{kHz}$. During the operation of the acoustic system, the geometric dimensions of the magnetostrictive material change, and it heats up, therefore, the acoustic system is equipped 
with a cooling system, which is represented by a tank 8, a pump 9, a cooling circuit 10 and pipelines. Items 4-10 are placed in module 2.

The very small droplets formed during acoustic dispersion are generally immobile, and the transfer to them is carried out by molecular diffusion. Laminar toroidal internal circulation arises in small and medium-sized droplets, which decreases the path length in the process of molecular diffusion.

To create the necessary circulating flows of liquid and gas at IV, V, VI stages of contact, increase the contact surface of the phases and merge the smallest drops of absorbent into the chamber 20 by the circulation pump 3 , a solution of the circulating absorbent is supplied through the pipeline The main part (90-70\%) of fresh absorbent is fed through the outlet. Further, a mixture of the smallest, medium and large droplets, together with the gas in a co-current mode, moves from top to bottom through the nozzle chamber 25. The condensation of the smallest droplets ensures $95 \%$ wettability of the loading surface. When the smallest drops come into contact with larger ones, they merge. Due to the developed wetted surface of the elements and the large surface of the liquid in their recesses, further diffusion of gas molecules into the liquid and chemical reactions occur. The liquid draining through the collection tray 16 into the drain collector enters the aeration columns 17 and, due to the appearance of vortex funnels, entrains the gas component with the smallest liquid droplets into the chamber 22. When gas and liquid move in the aeration columns 17, the rise of a large number of gas bubbles continues the process of mass transfer and the merging of the smallest drops. The depleted gas passing through the bubbling chamber is concentrated in the interchamber space of the V and VI stages. To regulate the partial pressure of the gas between the circuit of the I, II, III stages of contact and the circuit IV, V, VI of the contact stages, as well as to enhance the effect of surface diffusion of gas into the liquid, an electromagnetic valve is provided on the outlet pipeline 26.

Mass transfer between phases is accompanied by thermal effects, usually exothermic. An increase in temperature has an adverse effect on equilibrium. The operating temperature of the installation is maintained by the air conditioner 4, which supplies refrigerant to the space between the heat-insulating wall 27 and the housing of the installation [9-11].

To control pumps, solenoid valves, an acoustic dispersion module, an air conditioner, an automatic control unit 5 is provided, which is a discrete automatic control system. It includes the following modules: computing, interface, switching, selector, analog-digital, digital-to-analog. When the automatic control unit is started, the operation of the computing module is diagnosed, then through the interface module, which operates in full duplex mode, the primary information sensors are supplied, after which the signal is removed from the sensors and transmitted to the analog-to-digital conversion module and then to the controller (test mode). In the normal mode of sensor readings, the system issues the appropriate permission to form all peripheral equipment 2 , $3,4,5,23$ at the rate for the local control systems. In the event of a malfunction, it issues a system error code and switches to a protected mode. After formation at the rates, the ultrasonic generator 2 and the water cooling circuit of magnetostrictive transducers located in the acoustic disperser 23 are launched. The local control system of the acoustic circuit $(2,23)$ sets the amplitude of ultrasonic vibrations equal to $15 \mu \mathrm{m}$ through the acoustic feedback sensor and then it is maintained by the automatic adjustment circuit frequency. Then the gas supply system is started, and the pressure sensor measures the effective values of the pressure in the pipeline 6 in front of the solenoid valve. When the set pressure value in the pipeline 6 is reached, the solenoid valve opens and the gas enters the ceramic dispersers 7 or Hartmann's ultrasonic whistles, which disperse the gas in the liquid of chambers 8 and 9. To ensure efficient operation of the system, the solenoid valve controlled by the system so that the pressure at the inlet of the Hartmann ultrasonic whistle is stable. After that, frequency controllers (inverters) are started, which control pumps 3 according to the readings of the pressure sensors and the given starting characteristics. To ensure maximum productivity of the gas absorption process, automatic control regulates the 
operation of irrigation systems for chambers 13 and 20. Complex ultrasonic volume measurement modules are used here as sensors for the quality of dispersion, which are included in the negative feedback loops of the pumps 3 After all the chambers have established the preset parameters of pressures and temperatures, the global control system begins to reach optimal operating modes according to the criterion of minimizing the reduced costs at maximum productivity[12].

The design of the proposed samples of industrial devices is recommended to be performed according to the above parameters. As an analogue, the obtained formulas can be used to determine mass transfer coefficient $\left(\mathrm{K}_{\mathrm{S}}\right)$ and oxidizing capacity of the columns $\left(\mathrm{OC}_{\mathrm{K}}\right)$ for the dissolution of air oxygen in water, given in Part I (subsection 3.3). The obtained coefficients of mass transfer and oxidizing capacity of columns dy 50 and $70 \mathrm{~mm}$ in the system aeration column - aeration tank at a column height above the liquid level of 2 $\mathrm{m}$ and depths 1;2;3 m and columns DN 37 in the system biofilter - aeration column aeration tank can be used as a basis for calculating devices.

\section{Synthesis of feed proteins at food enterprises}

Liquid waste from food industry enterprises contains various valuable organic substances of natural origin, the reuse of which is of considerable interest. The problem of isolating valuable organic substances from waste of food industry enterprises is solved by biotechnological methods of using organic substances as substrates for the cultivation of certain microorganisms with the aim of accumulating biomass, enzymes, vitamins, etc. additives and other biologically active substances. Natural biological treatment is a traditional way of processing waste from the food industry. Part of the organic matter is deposited in sedimentation tanks of various designs, from where the more or less compacted sludge is taken out and used as fertilizer. The liquid is exposed to long-term aging in ponds, and then discharged into water bodies or directly used for irrigation. These are extensive methods that require the allotment of significant land areas and do not guarantee the safety of water bodies from pollution by drainage waters. The use of liquid waste in agricultural irrigated fields is also associated with a significant need for suitable land areas and expensive irrigation networks. In recent years, obtaining new types of energy and fuel has become a priority task for all countries of the world. Bioethanol (ethanol), one of the main types of biofuels, is obtained from sugar beets, sugarcane, corn, cereals, potatoes and other types of carbonaceous plant substrates. The global potential demand for bioethanol is 2 billion tons per year. Currently, the world produces 32 million tons of ethanol: of which 20 million tons - fuel ethanol, 8 million tons - ethanol for the chemical industry, 4 million tons - for the food industry. Chemical synthesis produces $7 \%$ ethanol, fermentation - 93\%. Obtaining alcohol by fermentation methods is accompanied by the formation of distillery bard during the production process. When alcohol (ethanol) is obtained from grain raw materials, more than $13 \mathrm{~m} 3$ per 1 ton of grain is formed. The problem of disposal of bard formed during the operation of factories producing alcohol is still an urgent problem. For example, a typical ethanol plant with a capacity of 42,000 dal / day. must utilize, process or sell about 4500-5000 tons of liquid bard daily. According to expert estimates, at present in Russia the annual volume of bard produced is about 700 million decaliters, of which no more than $5 \%$ is processed. Most enterprises are trying to drain the unclaimed bard into nearby water bodies or fields, which seriously worsens the ecological situation around distilleries. The problem is compounded by the fact that bard is a perishable liquid (humidity is about $80 \%$ ) and at a temperature of $15-30{ }^{\circ} \mathrm{C}$ it becomes contaminated with foreign microflora within a few hours. There are three main technological schemes that are used in the processing of bard: - schemes with evaporation 
stations to obtain "Dry bard (DDGS)"; - schemes with biogas production; - schemes for obtaining feed yeast. The first group - schemes with evaporation plants to obtain "Dry bard (DDGS)". The technology of "centrate evaporation" in evaporation plants is the most widespread in the world. This technology is offered, for example, by the Swedish company Alfa-Laval, the Danish company Atlas-Stord, a number of Chinese and Russian companies (the so-called "Chinese scheme"), etc. However, the cost of evaporation plants and, accordingly, all equipment for disposal is quite high, the evaporation process requires significant energy costs. Environmental problems are not fully resolved, because for disposal of "vapor" stationary treatment facilities are required. At the same time, serial equipment is produced for this technology. In Russia, the full cycle of bard processing in DDGS, according to our data, has been implemented only at one distillery (Spirtzavod Buinskiy Tatspirtprom). At a number of distilleries (Urzhumsky, Korystovo, etc.), a truncated cycle of processing bard into DDG has been implemented. In this case, only the solid phase of the bard - "cake" is processed, and the "centrate" is discharged (data on its utilization is not available). All this negatively affects the cost of the finished product - dry bard (DDGS), the main disadvantage of which is the imbalance of the resulting product in terms of crude protein, since its maximum content in dry bard does not exceed $24 \%$. Also, the high fiber content in dry bard - up to $20 \%$, significantly limits the range of its consumers. The second group is presented by schemes with biogas production. Currently, these schemes have not found wide application either abroad or in Russia. The technology for processing bard into biogas is based on anaerobic fermentation (fermentation without oxygen). Bard is served in special containers, into which anaerobic bacteria are introduced. The bacteria, eating the nutrients contained in the bard, produce biogas. Biogas can be utilized in plant boiler houses, and the precipitated sludge can be used as fertilizer. The advantage of this processing method is the relative low operating costs. However, this method of processing bard requires huge digesters (and hence, significant land plots), since the process of processing bard by anaerobic bacteria is extremely slow [13]. Another disadvantage of the method is a very long period of reaching the regimen - up to 6 months. And in our climatic conditions in the winter, all the gas produced is consumed to maintain the fermentation process. The third group combines technologies that provide for the preliminary utilization of sugar bard sugars by cultivating feed yeast on it. Production of protein-vitamin fodder yeast on distillery bard has been carried out for a long time and is considered a well-mastered process. In Russia, a number of plants for the production of dry feed yeast, operating on distillery bard, have been built (Beregovskaya, Mariinsky, Mamadyshsky, etc.). Feed yeast is a highly effective protein feed additive used in many agricultural enterprises and feed mills. The protein content in feed yeast can reach up to $50 \%$ or more. However, all such enterprises use highly inefficient equipment, which requires huge energy resources and serious operating costs. An example of such equipment is spray dryers using heated air as a heat carrier. Thus, a drying unit (one of two installed at the Beregovskaya plant) with a capacity of 10-15 tons / day for the finished product has a thermal capacity of 7-12 Gcal / h, consumes $1050 \mathrm{~m}^{3} / \mathrm{h}$ of natural gas and pumps 180,000 $\mathrm{m}^{3} / \mathrm{h}$ of air. Electricity consumption by the drying plant is $160-170 \mathrm{kWh}$. Such costs do not allow the company to have high profitability. Almost all income from the sale of products goes to pay for energy resources, while the situation is getting worse every year. It should also be noted that in this production yeast assimilates only $20-30 \%$ of all dissolved organic substances from bard, although theoretically all dissolved organic substances are potentially assimilable, since they are all of natural origin. In principle, it is impossible to assimilate all organic matter of bard when using yeast-like fungi as producers due to their cultural and physiological characteristics (only a limited set of organic substances has the ability to assimilate). But such a problem can be solved using bacterial associations, an example of which is biological wastewater treatment, where deep removal of a wide variety of organic 
substances is achieved precisely when using mainly bacterial associations cultivated in aerotanks (the so-called activated sludge). At the same time, numerous studies have shown that bacterial biomass, and even activated sludge from biological wastewater treatment plants, is no less effective when used as protein-vitamin feed additives in animal rations than fodder yeast. Thus, to increase the production of stillage production, one should abandon the use of yeast-like fungi as producers and switch to the use of bacterial associations. Such associations are easy to obtain in Combined Facilities (CF) of a special design, which have signs of biofilters and sedimentation aeration tanks. The production of fodder proteins at food industry enterprises is based on the experience of design, construction and operation of sewage treatment plants for the confectionery department. The technological scheme of purification of concentrated wastewater with a flow rate of $200 \mathrm{~m}^{3} /$ day, pollution concentrations according to BODp $1200-1600 \mathrm{mgO}_{2} / \mathrm{d} \mathrm{m}^{3}$, suspended solids - 1500-1800 mg / $\mathrm{dm} \mathrm{m}^{3}$, fats - $300 \mathrm{mg} / \mathrm{d} \mathrm{m}^{3}$, and photographs of structures are shown in Fig. 6 and 7.

After the grease traps, the production water of the enterprise flows by gravity into the sewage pumping station, from where it is pumped into the treatment plant unit $[14,15]$.

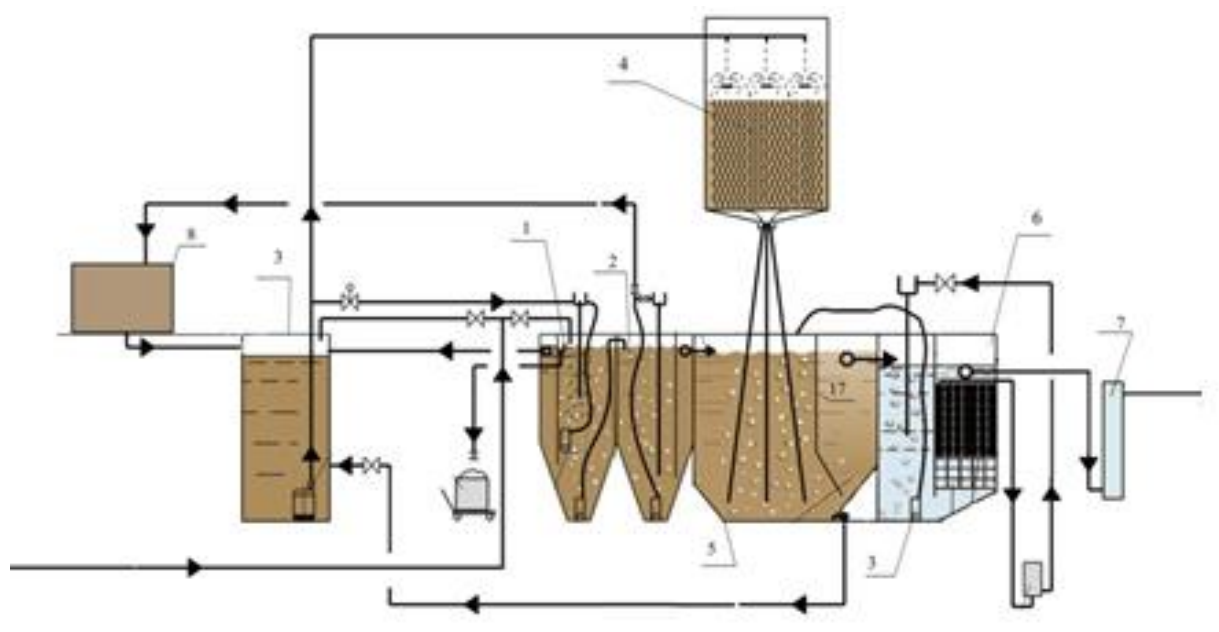

Fig. 6. Technological diagram.

Liquid waste is first fed into biocoagulators (1), where liquid with excess activated sludge (sludge dose 4-6 g / $\mathrm{d} \mathrm{m}^{3}$ ) is also automatically supplied from combined facilities (see Fig. 6). In biocoagulators, sedimentation of suspended solids occurs and, due to flotation, separation of fats (effect up to $70 \%$ ), as well as removal of up to $30 \%$ of suspended and dissolved organic compounds due to the sorption properties of the removed active biomass. 


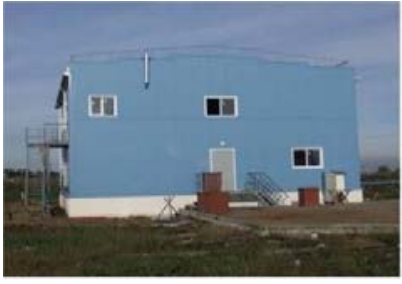

USC Building

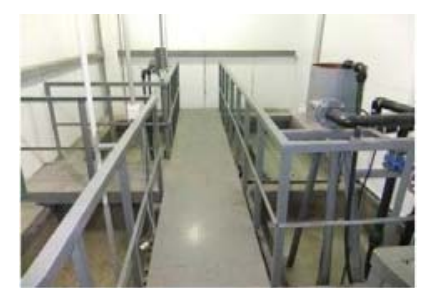

The biocoagulator and sludge thickener

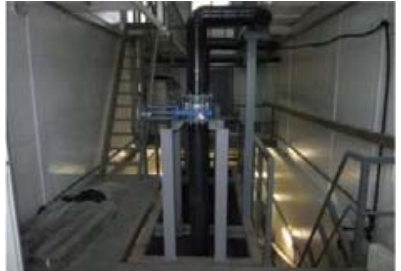

Displacement chamber with circulation pumps

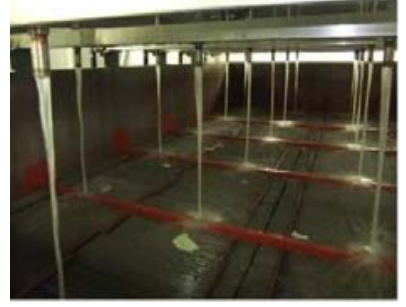

Biofilter

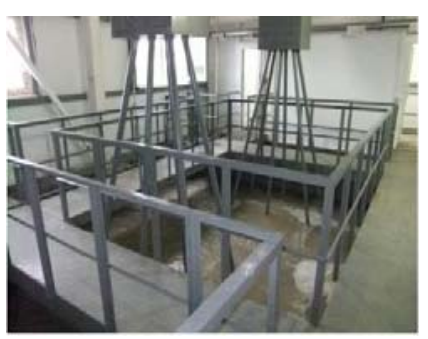

Aeration tank, settling tank

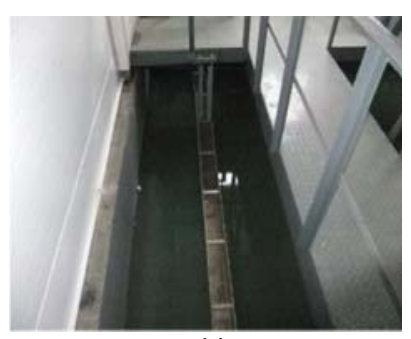

Post-treatment bioreactor

Fig. 7. Sewage treatment facilities of the confectionery shop.

Liquid and excess sludge (through a solenoid valve installed on the circulating liquid outlet pipeline) enter the receiving chamber of the water jet aerator installed in the biocoagulator (1). In the biocoagulator, the columns are lowered into the flocculation chamber, where the activated sludge comes into contact with the incoming liquid. The duration of contact of sludge with water in the flocculation chamber is 60 minutes. By lowering the temperature and flotation, the residual fat floats up. From the flocculation chamber, the sludge mixture through an expansion cone enters the settling zone (where a suspended sediment layer is formed) and phase separation occurs. The settled water is discharged through trays to the combined facilities. A mixture of sludge and sediment is deposited in the sludge.

\section{Economic efficiency of obtaining feed proteins}

To assess the economic feasibility of obtaining feed proteins from liquid waste of food industry enterprises, the cost of obtaining $1 \mathrm{~kg}$ of protein (dry matter) and the mass of the resulting product were estimated.

When calculating the mass of the produced product, the coefficient of the daily biomass growth from the mass of the initial organic substances was taken to be 0.4 and the protein content in the cultural biomass was $45 \%$. The addition of nutrients increases the protein gain by $15-30 \%$. For the calculation, a factor of 1.2 was adopted. The minimum range of application of biochemical plants for protein production begins with a volume of liquid waste of at least $200 \mathrm{~m}^{3} /$ day. and the concentration of organic substances according to 
BODp is $2000 \mathrm{mg} / \mathrm{dm}^{3}$, i.e. the mass of the obtained protein should be within $70 \mathrm{~kg} /$ day. At the same time, it is necessary to provide for the possibility of using the obtained biomass with a moisture content of $80 \%$ in feed mills (workshops) without drying and packaging.

\section{References}

1. V.P. Kolesnikov, D.V. Kolesnikov, Patent of China No. ZL201110069244.1 (2011)

2. V.P. Kolesnikov, D.V. Kolesnikov, Eurasian Patent № 023425. Installation for deep biochemical wastewater treatment. CO2 F3/02 (2016)

3. V. H. Núñez-Benítez, A. Barreras, A. Estrada-Angulo, et al., Livestock Scince, 243, $104373(2021)$

4. J. Wu, X. Zhang, R. Wang, et al., Animal Feed Science and Technology, 270, 114684 (2020)

5. V. Handa, D. Sharma, A. Kaur, et al., Biocatalysis and Agricultural Biotechnology, 25, 101600 (2020) doi.org/10.1016/j.bcab.2020.101600

6. L. Pinotti, A. Luciano, M. Ottoboni, et al., Journal of Cleaner Production, 294, 126290 (2021) doi.org/10.1016/j.jclepro.2021.126290

7. Y. Liu, X. Dong, B. Wang, et al., available online (2020) doi.org/10.1016/j.cjche.2020.11.014

8. M. Santamaría-Fernández, Mette Lübeck, Animal Feed Science and Technology, 268, 114605 (2020) doi.org/10.1016/j.anifeedsci.2020.114605

9. E.B.Abbade, World Development, 134, 105038 (2020) doi.org/10.1016/j.worlddev.2020.105038

10. Y. Etemadian, V. Ghaemi, A. R. Shaviklo, et al., Journal of Cleaner Production, 278, 123219 (2021) doi.org/10.1016/j.jclepro.2020.123219

11. F. Muneer, H. P. Hovmalm, S.-E. Svensson, et al., Journal of Cleaner Production, 294, 126304 (2021) doi.org/10.1016/j.jclepro.2021.126304

12. S. Despoudi, C. Bucatariu, S. Otles, et al., Food Waste Recovery (Second Edition), Processing Technologies, Industrial Techniques, and Applications, 3-19 (2021) doi.org/10.1016/B978-0-12-820563-1.00008-1

13. J. Seaberg, S. Kaabipour, S. Hemmati, et al., European Journal of Pharmaceutics and Biopharmaceutics, 154, 127-135 (2020) doi.org/10.1016/j.ejpb.2020.07.006

14. H. Yuan, L. Tan, K. Kida, et al., Journal of Bioscience and Bioengineering, available online (2021) doi.org/10.1016/j.jbiosc.2020.12.015

15. M. Morales, A. Arvesen, F. Cherubini, Bioresource Technology, 328, 124833 (2021) doi.org/10.1016/j.biortech.2021.124833 